\title{
Research on the Facade Image of the Goddess Hathor in Ancient Egypt
}

\author{
He Huang \\ Department of History, Peking University, Beijing, China \\ dorishh96@163.com
}

Keywords: Ancient Egypt, Hathor, Facade Image, traits and power.

\begin{abstract}
In the image system of ancient Egypt, Hathor is one of the very few gods that shown up on the front, she is also powerful and plentiful moral. Thus it is great significance to research on the facade image of the Goddess Hathor. This paper aims at starting with a few different situations that appeared on the front of the Hathor, to analyze the two sides of her character and strong power to elaborate her position of the world view in ancient Egypt and communication between the front image and viewer, therefore, a more reasonable explanation is given by this paper for the appearance of the front image of Hathor.
\end{abstract}

\section{古埃及哈托尔女神正面示人形象研究}

\author{
黄菏 \\ 北京大学历史系, 北京, 中国 \\ dorishh96@163.com
}

关键词：古埃及; 哈托尔; 正面像; 两面性; 动力

中文摘要. 在古埃及图像体系中, 哈托尔是极少数以正面示人的神祇之一, 而她自身又是一 位力量强大、寓意丰富的女神, 因此, 对哈托尔正面像的研究就具有重要意义。本文旨在从 哈托尔正面像出现的几种不同情况入手, 结合哈托尔自身性格的两面性与强大力量, 联系她 在古埃及世界观中的地位, 以及正面像与观者的交流作用, 对哈托尔正面像的出现给出一个 较为合理的解释。

\section{1. 引言}

在除雕塑之外的古埃及艺术表现形式里，人物大多以侧面正身示人，而哈托尔（Hathor） 女神则是为数不多的以正面示人的神祇中最主要的一位。哈托尔女神是古埃及神话中执掌欢 愉、性爱、生殖的女神, 她本身被赋予多重职责与含义, 她的正脸被抽象为一个强有力的符 号, 主要出现在叉铃（sistrum）、神庙柱头、镜子手柄等地方。因此, 基于哈托尔女神自身 强大的力量以及她在图像中具有的独特表现形式, 对于她的正脸形象的研究就具有重要意义。

此前, 虽然学界对哈托尔正面示人的原因并未给出一致的解释, 但已经涌现出大量对哈 托尔女神的研究著作, 对她的正面像也做出了一些猜想。比如罗伯茨 (Roberts, 1997) 在《哈 托尔的升起》（Hathor Rising: The Power of the Goddess in Ancient Egypt）一书中，对哈托尔 女神相关形象与仪式的发展及演变过程作出了详细介绍, 并提到了哈托尔的两面性, 认为哈 托尔正面示人的形象与她的两面性有关; 而布里克 (Bleeker,1973) 在《哈托尔和托特》 (Hathor and Thoth: Two Key Figures of the Ancient Egyptian Religion) 一书中, 将哈托尔与托特 (Thoth) 放在一起进行了讨论, 认为哈托尔相对于托特象征的知识与秩序, 表现了古埃及宗教循环中 
动力、强劲的一面, 而且对于哈托尔的正面像, 他认为这种形象的原型可以追溯到史前时期 部落酋长墓前悬挂牛头的木杆（C. J. Bleeker, 1973），猜测后来神庙中刻有哈托尔正脸形象 的石柱可能与此有关, 但对其发展过程及原因也并未作出明确解释。当然, 关于这个问题学 界还有其他不同观点, 但是这些观点也都基于大量的猜测与推理, 并没有得到普遍的认同。

本文将首先结合相关文献，说明 “正面” 形象在古埃及人观念中的特殊意义；然后，归 纳哈托尔女神正面像出现的几种不同情况及其历史演变过程, 并尝试结合哈托尔女神的相关 神话及自身特性, 对这几种情况分别作出初步的阐释; 最后, 将结合对古埃及宗教体系的认 识, 对为什么选择哈托尔这位女神进行正面描绘这一现象, 给出较为合理的解释。

\section{2. “正脸” 形象在古埃及传统中的特殊意义}

在古埃及的艺术传统中，除了三维立体的雕塑以外，人物的表现都遵从侧面正身率，即 人物的面部都表现为从侧面看到的样子, 而正脸则极少出现。对埃及人来说, 如果观者面对 一个形象的 “正脸” ，则意味着观者与形象直接交流并建立联系，因此观者会受到这个形象 的力量的直接影响。

“正脸” 形象的力量, 首先是可以直接对观者施加威慑和攻击的作用。比如，与神的正 脸直接接触, 就是十分危险的。在一些画面里, 当普通人出现在法老面前时, 会举起双手遮 在脸前, 借此避开不看法老的脸。这是因为法老被认为是太阳的化身，他的力量可以击倒所 有未受保护的人 (Alison Roberts, 1997) , 因此人们必须避开法老的正脸以躲避他的神力。再 比如在金字塔铭文中，一个恶魔就自夸道， “我的脸所朝向的人，他的脑袋和脖子就分了家”

（K. Sethe，1908-1922），也就是说他的脸所正对的地方都在他的力量的威胁之下。灵气 （sekhem）这种 “力量” 也被认为是从脸上辐射出来（Alison Roberts, 1997），这显然也与作 为力量、战争象征的塞赫麦特 (sekhmet) 女神的名字有着密切的联系。而塞赫麦特女神又与 哈托尔女神常常作为一个合一的形象出现, 我们由此可以猜测, 哈托尔的正面像在一定程度 上也具有这种震慑性的威力。

另一方面，这种 “正脸” 的形象也可以传递对观者有益的力量。比如一段对法老的赞美 诗中写道, “把你的脸朝向我, 啊, 升起的太阳”（Assmann, 1975），显然太阳神的化身一 法老, 也可以通过他的正脸, 赐予他所面向的人们以恩泽与活力。而在很多对神灵的祷告中, 也经常会出现与 “请把你仁慈的面庞朝向我” 类似的句子（Alison Roberts, 1997）。而哈托尔 的正面像也具有这样的积极有益的力量, 这一点将在后文中进一步说明。

因此，“正脸” 的形象实际上具有双重的力量：它既可以是危险的，用以直接威慑与攻 击对方; 又可以是有益的, 用以直接赐予对方生命与力量, 总之它所强调的是脸部的力量与 观者的直接交流互动。不过, 埃及人为了保护自己免于这种可能的威慑力的伤害, 除了一些 “正脸” 力量需要特别强调的地方，就极少在图像中刻画正脸的形象，而只是在文字中加以 描述。而哈托尔女神以正面的形象出现, 推测应该也有这两方面的含义, 即她应该是既有威 慑的作用, 也具有积极的力量, 这一点将在后文中结合哈托尔的自身特性进一步解释。

\section{3. 针对几种不同情况下哈托尔正面像的解读}

通过观察图像可以发现, 哈托尔在古埃及二维绘画、浮雕的体系中，大多数时候还是以 侧面正身的女神或母牛的形态出现, 符合于其他神在艺术中的表现形式。而当她以正面出现 的时候, 她的面部实际上都被表现为一种较为固定的形态, 即哈托尔女神特有的假发加上母 牛的耳朵。值得注意的是, 这些正面像都只刻画了女神的头部, 而并没有脖子以下的部分, 不同于以全身出现的合于侧面正身率的哈托尔形像。

所以, 正面的哈托尔女神像, 其实更加类似于一个抽象出来的符号, 相较于侧面全身像, 被赋予了更多的象征内涵。而这种象征符号, 大多出现在哈托尔女神的叉铃以及神庙柱头等 
几个固定的位置, 显然就是要配合这几种不同的语境, 表达各自的意义。下面, 我们就结合 哈托尔女神的自身特点, 针对这几种正面像出现的不同情况进行各自的分析。

\section{1 叉铃上的哈托尔正面像}

哈托尔正面像最常出现在叉铃柄部, 而且, 神庙柱头上的哈托尔正面像以及其他不同的 例子, 也基本上都是以此为原型进行的变形。因此, 对叉铃以及其上哈托尔正面像的研究就 十分重要。下面, 本文介绍叉铃的几种形制, 并归纳其出现的场景和发挥的作用, 再结合哈 托尔女神的特性以及正面像的出现对之进行解释说明。

3.1 .1 叉铃的形制分类及其演变

在古埃及早期历史中，哈托尔女神自己拿着这个乐器，因为毕竟她是掌管音乐的女神， 而在埃及后期, 叉铃则变成了一个和伊西斯的仪式有关系的更为流行的乐器。而在新王国之 前, 叉铃已经经过了长期的演化, 最终固定为两种标准的样式一一神殿状叉铃（naos sistrum） 和环状叉铃（loop sistrum）（Alison Roberts, 1997）。

（1）神殿状叉铃。这种样式的叉铃与神庙中的神秘祭仪相关，经常作为一个强有力的抚 慰性的乐器, 出现在女神或着是王后手中。它的上部类似于一个神庙最深处、用以盛放神灵 雕像的神殿，而乌赖乌斯则被雕刻在进入这个 “神殿” 的门口，正如同乌赖乌斯蛇守卫着的 神的居所。而用来制造声音的棒子和小圆盘, 则被插入上部的构造中, 尽管在浮雕和壁画中, 它们大多没有被表现出来。在柄和上层构造之间, 就是哈托尔的正面像, 仍然保留了哈托尔 特有的假发以及牛耳，但没有牛角和牛角之间的太阳圆盘。

（2）环状叉铃。戴有花环的环状叉铃, 是新王国时期常出现的一种叉铃样式, 多在神庙 里的女吟唱者颂歌时使用。与前一种唯一的不同是, 它的上部没有精心雕刻的神殿, 取而代 之的是一个简化的椭圆环。

\subsection{2 叉铃出现的场景及其作用}

按照叉铃的功能，可以把它分为两类：一种是叫做zsst的叉铃，被作为一个用来产生声音 的乐器, 通过声音去平息神灵的怒气; 另一种是叫做 shm的叉铃, 它是一个类似于权杖 (sceptre) 的器具, 特别是很类似于sekhem-sceptre, 象征着生命与力量 (Alison Roberts, 1984)。与此相 对, 也就出现了以下两种不同语境中, 叉铃发挥的不同作用, 一种是用来平息神灵的暴怒, 一种是作为传递强大神力的媒介。

（1）平息神的愤怒。叉铃主要的作用，是用来控制被激怒的神祇的愤怒与暴力，这与它 本身就是一个愉人心灵的乐器有关。有时, 叉铃也被作为一个礼物送给人们, 当做是生命的 象征。在辛努海的故事里, 皇室的孩子们把他们的叉铃献给法老, 这样他的情绪就可以被安 抚（Miriam Lichtheim, 2006）。更多的情况，则是它被用来平息哈托尔女神的怒意。

例如，在哈托尔的丹德拉（Dendera）神庙，就有对法老把叉铃献给哈托尔的描写，这种 礼物意味着 “去平息她的愤怒”（A. Mariette, Denderah, 1875）。叉铃也被用于祭司演奏, 用 以平息哈托尔的暴怒（ E. Chassinat \& F. Daumas, 1972）：

“强大的神的环状叉铃啊

驱赶走你的暴力,

你的卡的神殿状叉铃啊

消除你的盛怒。”

与此类似, 在一段Edfu神庙上的铭文中, “众神为她（哈托尔）摇动叉铃, 女神们为她 跳舞来赶走她的坏脾气”（C. DE Wit, 1951）。

那么, 为什么哈托尔女神总是会表现为如此易怒暴躁的形象呢? 古埃及人又为什么要选 择摇动叉铃作为平息她怒意的手段呢? 说到哈托尔的暴怒形象, 就不得不提到 “拉神之眼” 的神话，它的出现时间很早，最早见于金字塔铭文中（Coffin Texts 331）。

哈托尔是 “拉神之眼”, 她被拉神派到前方去寻找刚刚诞生的舒 (shu) 和泰芙努特 (Tefnut), 或者在另一些版本中被说成是去击倒反拉神的敌人。当她回到拉神面前时, 发现另一只眼睛 
取代了自己的位置, 被激怒的她离开了拉。最后, 在托特的劝解下, 哈托尔与拉和解回到拉 神身上。她在拉神的头顶上重新拥有荣耀的地位, 并化身为乌赖乌斯蛇的形态保护拉神 ( R. T. Rundle Clark, 1998）。

这个神话也可以与泰芙努特联系起来，泰芙努特是来自于努比亚的沙漠的凶残的狮子， 它的暴怒被托特和舒平息, 然后被引诱到埃及的土地, 在这里她变形为一个愉悦的仁慈的女 神的形象。而泰芙努特也常被描述为 “拉神之眼” （Richard H. Wilkinson, 2003），这就与哈 托尔被托特劝解回到拉神身上的情节相似。同样以 “拉神之眼” 的别称出现的还有塞赫麦特, 她和哈托尔都被描述为拉神的女儿，而塞赫麦特本身则意味着 “强有力的” (Richard H. Wilkinson, 2003），这也与神话中暴怒报复的眼睛形象相符。从 “拉神之眼” 和暴怒的形象 的角度来说, 这三位女神可以被看做是同一个神话形象。而她们共有的这种好战的性格, 应 该是哈托尔的一种天性, 是她易怒的暴脾气的一个体现。

故事最后出现的盘绕在拉神头顶的乌赖乌斯蛇，与叉铃上部守卫在神殿门口的乌赖乌斯 蛇的形象一致。联系前文提到把乌赖乌斯描述为 “燃烧着的火焰” 的例子, 我们可以推断, 从拉神之眼的神话中, 乌赖乌斯从愤怒的眼睛中继承到了暴怒与攻击性的力量, 之后又被作 为守护者出现在神与国王头顶和神殿门口等地方。而这种直接的强力的攻击性, 在叉铃上部 的和哈托尔一样正对前方的蛇的形象中, 得到了很好的阐释。这里正面的刻画, 显然是为了 让它的威慑与攻击能力得到更好的体现。

这样，哈托尔作为一个暴怒的好战的女神的形象，就被鲜活地呈现在我们面前。而这种 强力的暴怒作为对稳定秩序的威胁、作为足以毁灭人类的力量, 显然是必须要被平息的。而 安抚女神的手段, 就是摇动作为哈托尔象征物的叉铃。联系动词 “sss” (使……沙沙作响) 与 “sss.t”（叉铃）, 可以推测叉铃应该是模仿这种沙沙作响的纸莎草的声音, 借此来安抚 女神的情绪（A. Herbmann, 1959）。

讨论了这些哈托尔的暴怒的问题, 我们还是要回答为什么哈托尔会以正面示人。实际上, 哈托尔的这种怒意与她的正面像的出现有很大关系, 最明显的就是我们前面讨论过的, 正脸 的艺术形式可以加强威慑力的表达, 并把这种攻击力量直接指向观者。但真正的解释并不这 么简单，原因在于哈托尔被安抚后不同于泰芙努特和塞赫麦特，她会表现出一种异常迷人的 状态, 而这种强大的吸引力与她原有的攻击力一起, 会构成一种与正脸表达相关的性格的两 面性。对于这一点, 我们必须要结合她吸引力的一面才能说明, 因此将放在后面的部分再作 进一步阐释。

（2）传递神的强大力量。叉铃也是强大力量的象征, 可以作为传递力量的媒介。此类中 较常出现的, 是国王被手执叉铃的女神引领的画面。而这种画面最早出现在19、20王朝的时 期, 比如, 在卡纳克 (Karnak) 神庙多柱厅西墙上的巨幅浮雕中, 伊西斯 (Isis) 摇动她手中 的叉铃, 把塞提一世 (Seti I ) 引领到阿蒙-拉 (Amun-Re) 面前, 她头顶的文字刻写道 (Alison Roberts, 1997）:

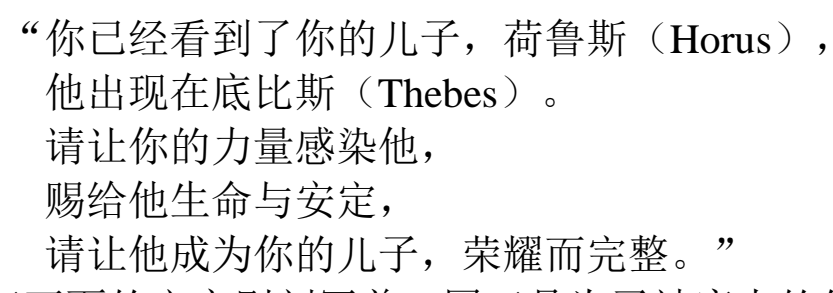

国王下面的文字则刻写着, 国王是为了神庙内的仪式而来, 他要见到他神圣的父亲、万 神之主, 为了得到 “生命与权利的永恒”。这里的女神虽然是伊西斯, 但她实际上是作为哈 托尔的一种同质转换出现。她成为了媒介, 让太阳神看到了法老, 借此传递给他生命力与统 治权。

值得注意的是，在伊西斯的叉铃中间，产生声音的棍子没有被画出来，但是哈托尔的正 面像以及头顶的乌赖乌斯被保留了下来。联系前文提到的，乌赖乌斯被描述为 “面朝敌人的 
脸上燃烧着的火焰”（P. Derchain, 1965），那么这里叉铃上的乌赖乌斯，就可以用来凸显叉 铃所象征的强大炽热的力量。另外, 柄部的哈托尔女神正面像, 应该也与前文提到的正脸的 巨大力量有关。而基于伊西斯在这里起着引领法老的作用，也可以推测其中的哈托尔正面像 在仪式中也具有引导性的作用，这种引导性，我们将在后文继续讨论。

一直延续到古典时代晚期, 很多学者都还提道了叉铃的巨大力量, 这也说明叉铃的象征 意义流传深远、影响很大。尤维纳利斯 (Juvenal) 告诉我们, 伊西斯可以运用她的叉铃让人 失明（Juvenal,1997）。普鲁塔克（Plutarch）也描绘了叉铃是如何凭借自己永恒运动的生命力 与创造力，击败了提丰（Typhon）（Griffiths, 1997）。

这种永不停息的生命力与创造力, 或许可以解释为与叉铃本身作为乐器而不断地被晃动 有关。不过, 叉铃既然还是哈托尔女神手中的象征物, 它的力量来源也应该是哈托尔本身, 而哈托尔作为掌管音乐的女神, 自然也被赋予了这种生机与活力。这种强大的动力, 也可以 推测是哈托尔以正面示人的原因之一，具体我们也将在后文再作解释。

\section{2 神庙柱头上的哈托尔正面像}

神庙的柱头也是哈托尔正面像最常出现的位置之一，而其形状也与神殿状叉铃相似，显 然是从神殿状叉铃变形演化而来。这种叉铃式的柱子一般出现在神庙的门廊入口处, 柱子的 最上部是乌赖乌斯蛇守护的神龛, 稍下处是哈托尔女神的正面雕像。比较典型的例子是巴哈 里（Deir el-bahari）的哈特谢普苏特（Hatshepsut）的神庙、丹德拉神庙以及菲莱（Philae）神 庙。

我们之前讨论的正面像的威慑作用，以及哈托尔本身怒意所包含的巨大力量，在柱头的 形象中也有表现。比如, 在贝尼哈桑 (Beni Hasan) 的斯庇欧斯・阿提米多斯 (Speos Artemidos) 神庙, 入口处的柱子上就有尚未完成的哈托尔的柱头。这个神庙是献给当地野蛮暴力的狮子 女神帕赫特 (Pakhet) 的，她被称为是 “抓咬的” ，和塞赫麦特与泰芙努特一样具有强大的 战斗力。在哈托尔的正脸上写着哈特谢普苏特的名字，而在入口上的铭文描写了希克索斯

(Hyksos）人入侵统治时的混乱, 并且赞扬了哈特谢普苏特如何重建统治、重申合法的继承 权。因此, 这里的哈托尔正面像, 有着斥退和希克索斯人一样的入侵的邪恶势力的作用, 这 里哈托尔的正面像表达了和帕赫特相符的强大力量, 维护了神庙的秩序。当观者走近神庙时, 首先看到的是高高在上的正对自己的女神之脸和拉神之眼, 受到了强大神力的直接威胁后必 然心生敬畏。我们还可以推断, 其他的几处神庙柱头, 至少表达了与此相同的威慑外人、守 护神庙的意义。

与叉铃所不同的是，柱头增加了与天空的联系，哈托尔女神的正面像，会在柱子的四个 面上都重复出现，这种面向四方的表现方式，显然表现了哈托尔与天空的紧密关系。哈托尔 的名字本身也体现了这一点，“哈托尔” 在古埃及语里写作 “ $\mathrm{Ht} \mathrm{Hr}$ ”，原意是 “荷鲁斯的居 所”。在这里，荷鲁斯指的是太阳神，而 “居所” 在古埃及常常是 “子宫” 的隐晦的表达。 所以实际上, 哈托尔的名字, 是要表达太阳从她这里诞生的意思。因此, 哈托尔实际上与地 平线的意义有关，她见证了太阳的新生。

以上部分，我们对哈托尔正面像出现的位置与功能作了回顾与总结。不能否认，哈托尔 的正脸都处在柱子、把柄中央这些需要高度对称的地方, 从实用的角度来说, 它的使用场景 决定了它只能以正面的形式出现。但是, 在古埃及的众神之中为何独独选择哈托尔女神进行 的描绘, 这仍然是一个值得讨论的问题。下面, 我们就结合哈托尔的自身特性和古埃及的神 话背景对之进一步讨论。 


\section{4. 进一步阐释哈托尔正面像出现的原因}

\section{1 从哈托尔的两面性和动力角度解释}

哈托尔最大的特点是她的两面性, 用布里克的话来说, 就是她是一个 “迷人而好战的” 形象（attractive-aggresive）。哈托尔两方面性格所带来的强大动力, 以及正面像所同时具有 的震慑人与勾人的特性, 共同带来了哈托尔正脸形象的出现。对于她迷人的一面, 也有重要 的作用，这在许多地方都有体现。

在荷鲁斯和赛特（Seth）的争斗的故事中，当巴巴（Baba）神的侮辱使他自尊受损而拒 绝继续工作时, 整个世界都进入了突然的停滞之中。这个时候拉的女儿哈托尔来到休息的拉 面前, 像一个孩子一样把自己的阴部展示给拉看, 这个玩闹的举动把拉逗笑了。之后拉站了 起来, 又坐回九神会面前, 让荷鲁斯和赛特继续为自己辩护（Lichtheim, 2006）。拉大笑的能 力在这里是一种对生命的肯定, 一种对周围世界的乐观积极的回应, 而在此之前, 他还刚刚 与世界疏离。哈托尔的甜言蜜语把拉哄回了众神面前, 让他可以继续之前被打断的荷鲁斯和 赛特的审判, 世界得以重新运行恢复生机。在这里, 哈托尔用她的吸引力诱惑拉, 带动了拉 重新工作的心情。这则神话中身体暴露的情节, 并不是为了给整个故事带来诙谐的插曲, 而 是真切地反映了古埃及的世界观与宗教观。

在古埃及人看来，是拉对他的女儿的爱激励着他每天穿过天空，哈托尔的鬼力正是推动 这个太阳循环的动力。没有她, 这里就没有日出。正是美丽年轻的哈托尔把与世隔绝高高在 上的统治者拉吸引到他俗世中来。女儿的热情与魅力消除了拉心中淡漠冷峻, 给拉带来愉悦 与欢乐, 激荡了他心灵中的活力, 前面讲过的叉铃也辅助带来了更大的心灵共鸣。在菲莱的 哈托尔神庙的赞美诗中，对此就有直接的描述（F. Daumas, 1968）：

“拉神永不停息地上升,

当他和他的女儿在一起时心灵愉悦，

他在他的天空中畅游, 万物和平，

他掉过头开始他的航行。”

然而在另外一些太阳的赞美诗里，拉又被描述为一个孩子，秘密地被他的母亲抚养并保 护，作为一个 “有鬼力的美丽年轻人” 在东方的地平线上升起。我们看到，哈托尔有时作为 太阳神的母亲出现, 有时又作为太阳神的女儿出现。这种奇怪的关系看似自相矛盾, 实则不 然。因为, 哈托尔的不同角色是出现在太阳神生命中的不同阶段的, 她以不同的方式帮助太 阳神完成生命循环的各个阶段。作为一个母亲，她给予了幼年的太阳神以诞生；作为妻子， 她辅佐了壮年的太阳神; 作为一个女儿, 他取悦老年的太阳神。最后, 在太阳神死后, 哈托 尔陪伴他进行死后的地下旅程, 直到他重生复活开始新一轮的生命循环 (G. Pinch, 2002)。

值得注意的是, 当太阳神经历出生、衰老、死亡的过程时, 不论是母亲、妻子还是女儿, 哈托尔始终都是以一个年轻而有鬼力的形象出现, 她似乎是永葆青春活力的, 以她的激情与 欢愉鼓舞着太阳神迎来一次次人生的新阶段。实际上，在太阳循环中，常被提到的玛阿特

(Maat) 女神是作为秩序象征指引这个过程的, 而哈托尔则是内在的力量, 推动了对生命与 存在的欲望（Alison Roberts, 1997）。哈托尔是这个循环中不变的动力, 始终表现为年轻充满 生命力的状态，一直给予了太阳神的循环以力量的支持，注入了活力。

哈托尔所具有的这种巨大动力也不难理解，因为她本身就是 “司掌音乐的女神”、“性 爱女神”与 “醉酒的女神” (Richard H. Wilkinson, 2003)，她常常表现为狂喜的、充满活力 与生机的样子。放荡不羁的醉酒、活泼诱人的音乐、意乱情迷的爱欲, 这些直抵人心的欢愉 与狂喜, 构成了她从内心深处影响人的强大力量, 撩拨着人们去运动、前行, 荡漾开人们内 心深处的渴望与激情。

从哈托尔前面提到的两面性的角度来看, 我们也可以很好地认识这种动力。一方面, 她 是作为拉神之眼出现的一个暴怒的形象; 另一方面, 她的恩宠和仁慈也是为人所称赞的, 她 是生气勃勃的。而对于这种骇人暴怒与诱人鬼力的合一, 我们还可以作出更进一步的解释。 
在埃及人自己的观念里, 哈托尔经常被和塞赫麦特和Bast放在一起比较。埃及人自己认为, “哈托尔和塞赫麦特一样愤怒，又和贝斯特（Bast）一样欢愉” （C. ED. Wit, 1951）。我们 前面讲叉铃的时候提到过，塞赫麦特是孟菲斯（Memphis）的尚武的、易怒的狮子女神，而 贝斯特是布巴斯提斯（Bubastis）的守护女神，更多地展现为快乐友善的形象而受人们喜爱。 与此类似的对哈托尔的描述，还有 “泰芙努特的美丽的形态”（H. Junker, 1917），泰芙努特 是和塞赫麦特一样的凶残的战争女神。我们看到, 哈托尔可以和泰芙努特与塞赫麦特一样被 激怒而展现出愤怒狂暴的一面，但是与她们不同的是，她的怒意一定会被平息，而为了确保 哈托尔的怒意被平息，埃及人每年都要在三角洲一带举行采纸草节( “sss wad.w n Ht Hr”， Plucking of the papyrus for Hathor)来取悦安抚女神（C. J. Bleeker, 1973）。而女神一消除怒意 之后, 就会展现出无人可比的诱人姿态, 作为生命中美好力量的给予者存在, 继续吸引着人 和神再次走向她。

她的这种动荡又不确定的性格本身，就展现出巨大的生机与活力，就像一个敢爱敢恨、 风情万种的少女, 带来了古埃及世界中不确定的却又是激荡的一面, 在 “愤怒一一被平息一 诱惑——愤怒” 的来回摆动中推动着世界的前行。而无论是斥退击倒人时的暴怒，还是诱人 前来时的美丽, 都具有着无人可比的强大力量, 这些力量本身也强化了哈托尔推动太阳循环 的动力。当然, 正因为哈托尔这种动力太过强大又难以控制、阴晴不定, 就需要玛阿特女神 时刻掌管世界的秩序, 确保太阳神的航行维持在正确的轨道上。

\section{2 从哈托尔观者与正面像与观者的联系的角度解释的关系}

至此, 我们已经论述了哈托尔的强大动力以及预计她对太阳循环的推进作用。那么, 这 些与她的正面像有什么关系呢?

首先，最直白的原因是哈托尔的双重震慑力和吸引力，可以同时通过正脸的形象得到很 好的表达, 正脸的形象通过与观者的直接交流, 既可以诱惑人前来, 又可以斥退敌人守护自 身。这一点我们在前面已经讨论。

更深层的原因，则是通过开放的正脸形象，让观者参与到哈托尔的动力循环中去。我们 都知道, 侧面的形象, 往往是以叙述的目的出现, 它们都有一个自己相对封闭的叙事空间, 而观者只能观看却不能进入这个空间与之发生联系。而正面的形象则可以和观者正面地交流, 这个空间是相对开放的, 观者因为可以和正脸发生直接的联系而被纳入了这个形象的叙事空 间之中。我们可以从这个角度去解释哈托尔的形象, 当她以侧面正身出现在叙事图像的整体 中时, 她是在与画面中的其他神祇和法老发生联系, 她就像一个轮子推动了这个世界的循环。 而当她以正面的形象出现时，观者看到了她的正脸而与她站在了同一个叙事空间里，她就是 与观者发生了联系。所以, 观者实际上是通过这种交流与联系, 被哈托尔吸引到了世界的周 期循环中去, 因此他自身的生命也借助哈托尔的力量的推动得到了重生于更新, 而他也可以 直接得到哈托尔给予的重生的力量。

因此，这种正脸的形象实际上是高度符号化的，它是一个联系观者与神祇力量的媒介， 可以直接传递生命与动力。这也就可以解释为什么哈托尔的正面像不与侧面像一样以完整的 全身形态出现, 因为这里只有头部的正面与观者发生了交流的作用, 而这种作用后来被高度 抽象化了，因此只有发生关系的面部作为一个象征符号被保留了下来。

\section{5. 结论}

综上所述, 我们就可以对哈托尔的正面像的出现, 作出一个较为合理的解释。哈托尔自 身所具有的暴怒与诱人的双重特性蕴含了巨大的力量, 这种力量既常常与观者发生联系起到 震慑或恩赐的作用, 又是推进太阳循环的一个强大动力。因此, 抽象化了的哈托尔正面像, 既可以作为媒介，直接与观者发生交流并传递力量；又可以与观者进入同一个空间，把观者 纳入到整个世界的周期循环中去, 赐予他们生命与动力。因此, 哈托尔的正面像也就经常作 
为一个强力的符号, 在神庙柱头、叉铃等需要力量来震慑或给予恩赐的情景中存在, 发挥自 己的独特作用。

\section{References}

[1] A. Herbmann, Altagyptische Liebesdichtung, 14-28, 1959, cited in Bleeker, C. J., Hathor and Thoth: Two Key Figures of the Ancient Egyptian Religion, Leiden, 1973.

[2] Alison Roberts, Cult Objects of Hathor: An Iconographic Study, chapter 2, Oxford University, 1984, cited in Roberts, Alison, Hathor Rising: The Power of the Goddess in Ancient Egypt, Rochester, 1997.

[3] A. Mariette, Denderah, II, 53 a, 1875, cited in Bleeker, C. J., Hathor and Thoth: Two Key Figures of the Ancient Egyptian Religion, Leiden, 1973.

[4] Assmann, Agyptische Hymnen und Gebete, 497 (No 240), Zurich and Munich, 1975, cited in Roberts, Alison, Hathor Rising: The Power of the Goddess in Ancient Egypt, Rochester, 1997.

[5] Bleeker, C. J., Hathor and Thoth: Two Key Figures of the Ancient Egyptian Religion, Leiden, 1973.

[6] C. DE Wit, Le role et le sens du lion dans l'Egypte ancienne, 306, 1951, cited in Bleeker, C. J., Hathor and Thoth: Two Key Figures of the Ancient Egyptian Religion, Leiden, 1973.

[7] Clark, R. T. Rundle, Myth and Symbol in Ancient Egypt, New York, 1998.

[8] E. Chassinat and F. Daumas, Le temple de Dendara 7, 91, Dario, 1972, cited in Roberts, Alison, Hathor Rising: The Power of the Goddess in Ancient Egypt, Rochester, 1997.

[9] F. Daumas, Zeitschrift für Ägyptische Sprache und Altertumskunde, 95, 1968, 12, \$20(II3-4), 13, cited in Roberts, Alison, Hathor Rising: The Power of the Goddess in Ancient Egypt, Rochester, 1997.

[10]Gillam, Robyn, Performance and Drama in Ancient Egypt, London, 2005.

[11]Graves-Brown, C., Dancing for Hathor: Women in Ancient Egypt, London, 2010.

[12] Griffiths(ed), Plutarch's de Iside et Osiride, 219, Lucan, 8, 832, Pharsalia, cited in Roberts, Alison, Hathor Rising: The Power of the Goddess in Ancient Egypt, Rochester, 1997.

[13]H. Junker, Die Onurislegende, 110, 1917, cited in Bleeker, C. J., Hathor and Thoth: Two Key Figures of the Ancient Egyptian Religion, Leiden, 1973.

[14]Juvenal, Saturae 13, 93, cited in Roberts, Alison, Hathor Rising: The Power of the Goddess in Ancient Egypt, Rochester, 1997.

[15]K. Sethe, Die altgyptischen Pyramidentexte, 4 vols., Leipzig, 1908-1922, PT§682e-f, cited in Roberts, Alison, Hathor Rising: The Power of the Goddess in Ancient Egypt, Rochester, 1997.

[16]Lichtheim, Miriam, Ancient Egyptian Literature, UC Press, 2006.

[17]P. Derchain, Le papyrus Salt 825 (B.M 10051), ritual pour la conservation, de la vie en Egypte, 142, XIII, 3, Brusseles, 1965, cited in Roberts, Alison, Hathor Rising: The Power of the Goddess in Ancient Egypt, Rochester, 1997.

[18]Pinch, G., Egyptian Mythology: A Guide to the Gods, Goddesses and Traditions of Ancient Egypt, Oxford, 2002.

[19]Redford, Donald B., The Oxford Encyclopedia of Ancient Egypt, Oxford, 2001. 
[20] Roberts, Alison, Hathor Rising: The Power of the Goddess in Ancient Egypt, Rochester, 1997. [21]Wilkinson, Richard H., The Complete Gods and Goddesses of Ancient Egypt, London, 2003. 